\title{
RA'EGA
}

O ESPACYO GEOGRÁFICO EM ANÁLISE

\section{VARIAÇÃO ESPACIAL E TEMPORAL DO SALDO DE RADIAÇÃO SUPERFICIAL EM UMA ÁREA DO SUL DO AMAZONAS, BRASIL}

\section{SPATIAL AND TEMPORAL VARIATION OF SURFACE RADIATION BALANCE IN AN AREA OF THE SOUTHERN AMAZONAS, BRAZIL}

Vagner Marques Pavão

Programa de Pós-Graduação em Física Ambiental / Instituto de Física Universidade Federal de Mato Grosso - UFMT

Cuiabá, MT, Brasil e-mail:vagnermpavao@gmail.com

Carlos Alexandre Santos Querino Sistema de Proteção da Amazônia Centro Regional de Porto Velho

Porto Velho, RO, Brasil e-mail: carlosquerino@gmail.com

Cristina Aparecida Beneditti Sistema de Proteção da Amazônia Centro Regional de Porto Velho

Porto Velho, RO, Brasil e-mail: crisengamb@gmail.com

Larissa Leite Pavão

Programa de Pós-Graduação em Física Ambiental/Instituto de Física Universidade Federal de Mato Grosso - UFMT

Cuiabá, MT, Brasil e-mail: lario.l@hotmail.com

Juliane Kayse Albuquerque da Silva Querino Instituto de Educação, Agricultura e Ambiente Universidade Federal do Amazonas - UFAM Manaus, AM, Brasil e-mail:julianekayse@hotmail.com

Nadja Gomes Machado Laboratório da Biologia da Conservação Instituto Federal de Mato Grosso - IFMT Cuiabá, MT, Brasil e-mail:nadja.machado@blv.ifmt.edu.br

Marcelo Sacardi Biudes Programa de Pós-Graduação em Física Ambiental/Instituto de Física Universidade Federal de Mato Grosso - UFMT Cuiabá, MT, Brasil e-mail:marcelo@fisica.ufmt.br 
PAVÃO, V. M.; QUERINO, C. A. S.; BENEDITTI, C. A.; PAVÃO, L. L.; QUERINO, J. K. A. S.; MACHADO, N. G.; BIUDES, M. S.

VARIAÇÃO ESPACIAL E TEMPORAL DO SALDO DE RADIAÇÃO SUPERFICIAL EM UMA ÁREA DO SUL DO AMAZONAS, BRASIL

Recebido em: 08/07/2015

Aceito em: 29/02/2016

\title{
Resumo
}

O sul do Amazônas apresenta diversas fisionomias vegetais naturais que estão sendo substituidas pela agropecuária. A variação espacial no balanço de radiação é fortemente relacionada à alterações no uso do solo. Sendo assim, o objetivo desse trabalho foi avaliar a variação espaço-temporal do saldo de radiação sobre diferentes superfícies no sul do estado do Amazonas por imagens Thematic Mapper (TM) Landsat 5. A área de estudo foi no município de Humaitá-AM, onde foi possível identificar área de floresta, campo limpo, campo sujo e área de cidade. As imagens foram adquiridas no site do U. S. Geological Survey referentes aos dias 15/07/2009 e 02/07/2010. O Índice de Vegetação da Diferença Normalizada (NDVI), o Índice de Vegetação Ajustado ao Solo (SAVI) e o Saldo de Radiação da superfície (Rn) foram calculados nas etapas intermediárias do SEBAL (Surface Energy Balance Algorithms for Land). Os maiores valores de NDVI e SAVI foram observados em áreas de floresta, seguido pelas áreas de campo sujo, campo limpo e cidade. O Rn relacionou positivamente com NDVI e SAVI. O Rn variou entre 550 e $565 \mathrm{~W} \mathrm{~m}^{-2}$ na floresta, 520 e $530 \mathrm{~W} \mathrm{~m}^{-2}$ nos campos limpo e sujo e abaixo de $500 \mathrm{~W} \mathrm{~m}^{-2}$ na área urbana de Humaitá. Diante disso, verificou-se que a densidade da vegetação é um fator determinante para a disponibilidade de energia no ambiente.

Palavras-chave: uso do solo; floresta amazônica; trocas de energia; interação solo-planta-atmosfera.

\begin{abstract}
The south of the Amazon has several natural vegetation types that are being replaced by agriculture. The spatial variation in the radiation balance is strongly related to land use change. Thus, the aim of this study was to evaluate the spatial and temporal variation of the net radiation on different surfaces in southern Amazonas state using Thematic Mapper (TM) Landsat 5 images. The study area was the Humaitá-AM municipal area where were identified forest, grassland, grassland with shrub and city area. The images were acquired on the site of the US Geological Survey referring to the days 15/07/2009 and 02/07/2010. The Normalized Difference Vegetation Index (NDVI), Adjusted Vegetation Index to Soil (SAVI) and the surface net radiation (Rn) were calculated in the intermediate stages of SEBAL (Surface Energy Balance Algorithms for Land). The highest values of NDVI and SAVI were observed in forest areas, followed by grassland, grassland with shrub and city areas. The Rn positively related with NDVI and SAVI. The Rn ranged between 550 and 565 $\mathrm{W} \mathrm{m} \mathrm{m}^{-2}$ in the forest, 520 and $530 \mathrm{~W} \mathrm{~m}^{-2}$ in grassland and grassland with shrub and below $500 \mathrm{~W} \mathrm{~m}^{-2}$ in the urban area of Humaitá. Therefore, it was found that the density of vegetation is a determining factor in the availability of energy in the environment.
\end{abstract}

Keywords: land use; Amazon forest; energy exchanges; soil-plant-atmosphere interaction. 
PAVÃO, V. M.; QUERINO, C. A. S.; BENEDITTI, C. A.; PAVÃO, L. L.; QUERINO, J. K. A. S.; MACHADO, N. G.; BIUDES, M. S.

VARIAÇÃO ESPACIAL E TEMPORAL DO SALDO DE RADIAÇÃO SUPERFICIAL EM UMA ÁREA DO SUL DO AMAZONAS, BRASIL

\section{INTRODUÇÃO}

O sul do estado do Amazonas contempla uma área de transição entre ecossistemas de floresta densa e campos naturais (limpo e sujo). Os campos constituem o complexo de campos e matas abertas dos "Campos Naturais de Humaitá-Puciari" que abrangem parte do sul do Amazonas e norte de Rondônia. Sua vegetação predominante são gramíneas e florestas circundantes formadas por florestas mesófilas baixas, florestas perinifólias altas, palmeiras, castanhais e outras (BRAUN e RAMOS, 1959; VIDOTTO et al., 2007).

Ao longo dos anos esses ambientes vêm sofrendo impactos em sua cobertura natural devido às atividades antrópicas como extrativismo de madeira, conversão de florestas em pastagem e grilagem de terras. Apesar do estado do Amazonas possuir o menor índice de área desmatada da Amazônia Legal, sua porção sul tem se tornado uma nova fronteira agropecuária, uma vez que a mesma tem apresentado as maiores frentes de desmatamento do estado (YANAl et al., 2010).

O mapeamento das áreas com diferentes tipos de cobertura vegetal pode ser realizado por meio de índices de vegetação estimados a partir de valores de refletâncias medidos por sensores orbitais. $O$ índice de vegetação mais popular é o Índice de Vegetação da Diferença Normalizada (Normalized Difference Vegetation Index - NDVI), o qual é capaz de determinar a densidade de fitomassa foliar fotossinteticamente ativa por unidade de área (DEMARCHI et al., 2011). Teoricamente, o NDVI pode variar entre -1 e +1 ; quanto mais próximo de +1 , o NDVI indica maior densidade de cobertura vegetal, ou seja, apresenta-se em estágio denso, úmido e bem desenvolvido. Por oturo lado, valores negativos indicam superfície com água.

A desvantagem do uso do NDVI está no fato deste ser altamente influenciado pelo brilho e pelo efeito espectral do solo, o que prejudica as avaliações sobre a vegetação (ELMORE et al., 2000). No entanto, o Índice de Vegetação Ajustado ao Solo (Soil-Adjusted Vegetation Índex - SAVI) é um índice que leva em consideração efeitos do solo exposto nas imagens analisadas, quando a superfície não esta completamente coberta pela 
PAVÃO, V. M.; QUERINO, C. A. S.; BENEDITTI, C. A.; PAVÃO, L. L.; QUERINO, J. K. A. S.; MACHADO, N. G.; BIUDES, M. S.

VARIAÇÃO ESPACIAL E TEMPORAL DO SALDO DE RADIAÇÃO SUPERFICIAL EM UMA ÁREA DO SUL DO AMAZONAS, BRASIL

vegetação (BORATTO e GOMIDE, 2013). O SAVI foi desenvolvido por Huete (1988) para minimizar os efeitos da exposição do solo na caracterização da vegetação. Para tanto, o SAVI leva em consideração o fator de ajuste para o substrato do dossel (L) que geralmente é usado o valor de 0,5 para cobertura vegetal intermediária e 0,25 para altas densidades de vegetação. Para casos em que L for igual a zero, o SAVI tem o mesmo valor do NDVI (HUETE, 1988).

A substituição de floresta em pastagem e área urbana alteram significativamente a temperatura e umidade relativa do ar e os balanços de radiação e energia da superfície em escala local e regional (BIUDES et al., 2012; 2015). As áreas de floresta possuem maior indice de vegetação, devido à maior biomassa, o que inflencia diretamente no balanço de radiação e, posteriormente no balanço de energia local, acarretando variação no particionamento de energia destinada para calor sensível, latente e aquecimento do solo (BIUDES et al., 2009; 2015).

Estudos envolvendo o balanço de radiação são realizados em sua grande maioria com o uso de saldo radiômetros instalados diretamente em campo. Contudo, essa metodologia apresenta limitação na espacialização das medidas em escala regional (GUSMÃO et al., 2012). Diante disso, o sensoriamento remoto tem se mostrado como ferramenta importante para estimatimar o saldo de radiação, visto que permite a obtenção de informações e suas variações espaço-temporal, com baixo custo e grande correlação com dados medidos em campo (SILVA et al., 2005; LIBERATTO et al., 2011; OLIVEIRA e MORAES, 2013; SANTOS et al., 2014).

Como existe uma lacuna no conhecimento sobre a variação espacial e temporal do saldo de radiação sobre diferentes uso do solo no sul do Amazonas, o objetivo desse trabalho foi avaliar a variação espaço-temporal do saldo de radiação sobre diferentes superfícies no sul do estado do Amazonas por imagens Thematic Mapper (TM) Landsat 5.

\section{MATERIAL E MÉTODOS}

\section{1. Área de estudo}

A área de estudo está localizada no município de Humaitá, no sul do 
PAVÃO, V. M.; QUERINO, C. A. S.; BENEDITTI, C. A.; PAVÃO, L. L.; QUERINO, J. K. A. S.; MACHADO, N. G.; BIUDES, M. S.

VARIAÇÃO ESPACIAL E TEMPORAL DO SALDO DE RADIAÇÃO SUPERFICIAL EM UMA ÁREA DO SUL DO AMAZONAS, BRASIL

estado do Amazonas, à margem esquerda do Rio Madeira, afluente do Rio Amazonas (Figura 1). O município de Humaitá está cerca de $200 \mathrm{~km}$ ao norte da capital de Rondônia, Porto Velho, e 675km ao sul de Manaus, capital do Amazonas. Seus vizinhos são os municípios de Manicoré ao Norte, Tapua ao Leste, Canutama a Oeste e o estado de Rondônia ao Sul (MARTINS et al., 2006).

Nesta região, existem três ecossistemas naturais adjacentes entre si: (I) floresta densa caracterizadas por vegetação de grande porte e pequeno espaçamento entre as espécies vegetais ali presentes; (II) campo sujo com vegetação de porte menor e menos densa, com gramíneas associadas à arbustos e árvores de 3 a 5 m de altura (CAMPOS, 2009); e (III) campo limpo, onde predomina vegetação rasteira (Figura 1).

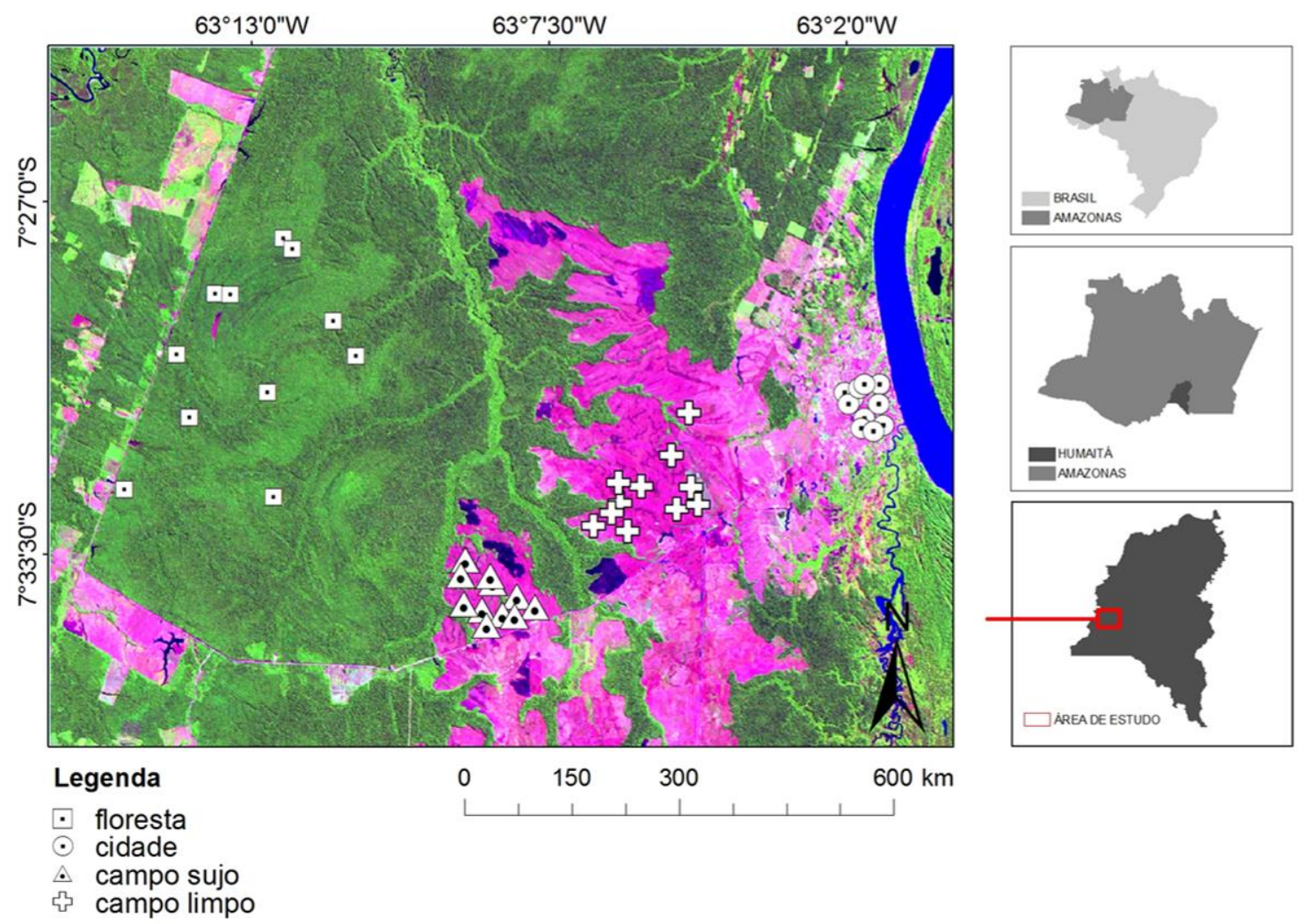

Figura 1: Localização da área de estudo no município de Humaitá, Amazonas, Brasil.

O clima da área de estudo é do tipo Am, segundo a classificação de Koppen, com temperatura média do ar variando entre de 25 e $27^{\circ} \mathrm{C}$, umidade relativa do ar em torno de $60 \%$ e precipitação anual média de $2500 \mathrm{~mm}$. A região apresenta um período chuvoso entre os meses de novembro e abril com 
PAVÃO, V. M.; QUERINO, C. A. S.; BENEDITTI, C. A.; PAVÃO, L. L.; QUERINO, J. K. A. S.; MACHADO, N. G.; BIUDES, M. S.

VARIAÇÃO ESPACIAL E TEMPORAL DO SALDO DE RADIAÇÃO SUPERFICIAL EM UMA ÁREA DO SUL DO AMAZONAS, BRASIL

totais pluviométricos mensais próximos a $400 \mathrm{~mm}$, e seco de pequena duração entre os meses de junho a setembro com precipitação variando de 0 a $100 \mathrm{~mm}$, sendo os meses de maio e outubro considerados como meses de transição (FERREIRA et al., 2005; CAMPOS et al., 2012).

\subsection{Aquisição e processamento das imagens}

Neste trabalho, foram utilizadas imagens do Thematic Mapper geradas em 15/07/2009 e 02/07/2010, a bordo do satélite Landsat 5, obtidas junto à $U$. S. Geological Survey (www.glovis.usgs.gov). Após a aquisição das imagens, elas foram recortadas e utilizadas para estimativa do saldo de radiação da superfície. As imagens adquiridas para esse trabalho referem-se à estação seca da área de estudo por facilitar a comparação espaço-temporal dos alvos na área de estudo. As imagens foram processadas com o uso do software ERDAS 2014 e os mapas foram elaborados no ArcGIS 10.3.

\subsection{Estimativa do Saldo de Radiação da Superfície}

O saldo de radiação foi estimado como etapa intermediária do Surface Energy Balance Algorithms for Land (SEBAL) que tem como etapa final a estimativa da evapotranspiração (BASTIAANSSEN et al., 2000). Os coeficientes de calibração mínimo e máximo e as irradiâncias espectrais no topo da atmosfera utilizados nesse trabalho foram baseados em Chander et al. (2007).

A calibração radiométrica (Equação 1) é a transformação dos níveis de cinza de cada pixel da imagem em radiância espectral monocromática $\left(L_{\lambda i}\right)$, que representa a energia solar refletida por cada pixel por unidade de área e tempo para cada comprimento de onda médio (MARKHAM e BAKER, 1987).

$$
\mathrm{L}_{\lambda \mathrm{i}}=\mathrm{a}_{\mathrm{i}}+\left(\frac{\mathrm{b}_{\mathrm{i}}-\mathrm{a}_{\mathrm{i}}}{255}\right) \mathrm{ND}
$$

em que a e b são as radiâncias mínimas e máximas; DN é o nível de cinza ou número digital do pixel (de 0 a 255) e i identifica a banda de 1 a 7 do sensor TM.

A reflectância monocromática $(\rho \lambda i)$ foi obtida a partir da razão entre 
PAVÃO, V. M.; QUERINO, C. A. S.; BENEDITTI, C. A.; PAVÃO, L. L.; QUERINO, J. K. A. S.; MACHADO, N. G.; BIUDES, M. S.

VARIAÇÃO ESPACIAL E TEMPORAL DO SALDO DE RADIAÇÃO SUPERFICIAL EM UMA ÁREA DO SUL DO AMAZONAS, BRASIL

radiação refletida e radiação incidente (Equação 2).

$\rho_{\lambda \mathrm{i}}=\frac{\pi \cdot L_{\lambda \mathrm{i}}}{\mathrm{k}_{\lambda \mathrm{i}} \cdot \cos Z \cdot \mathrm{d}_{\mathrm{r}}}$

em que $L_{\lambda i}$ é a radiância espectral de cada banda, $k_{\lambda i}$ é a irradiância solar espectral de cada banda no topo da atmosfera $\left(\mathrm{W} \mathrm{m}^{-2} \mu \mathrm{m}^{-1}\right), \mathrm{Z}$ é o ângulo zenital solar (Equação 4) e dr é o inverso do quadrado da distância relativa Terra-Sol (Equação 3), dada por lqbal (1983).

$\mathrm{dr}=1+0,033 \operatorname{Cos}\left(\frac{\mathrm{Dj} \cdot 2 \pi}{365}\right)$

em que DJ representa o dia Juliano, ou seja, o número sequencial de determinado dia no ano.

$\operatorname{Cos} \mathrm{Z}=\operatorname{Cos}\left(\frac{\pi}{2}-\mathrm{E}\right)$

em que $\mathrm{E}$ é o ângulo de elevação do sol, disponível no metadado de cada imagem obtida.

O albedo planetário $\left(\alpha_{\text {toa }}\right)$ é aquele não ajustado ao efeito da atmosfera terrestre, e foi obtido pela combinação linear das refletâncias monocromáticas (Equação 5).

$\alpha_{\text {toa }}=0,293 \rho_{1}+0,274 \rho_{2}+0,233 \rho_{3}+0,157 \rho_{4}+0,033 \rho_{5}+0,011 \rho_{7}$

em que $\rho_{1}, \rho_{2}, \rho_{3}, \rho_{4}, \rho_{5}$ e $\rho_{7}$ são os albedos planetários das bandas $1,2,3,4,5$ e 7 do TM Landsat 5.

O albedo da superfície ou albedo corrigido para efeitos atmosféricos ( $\alpha$ ) foi obtido pela Equação (6).

$\alpha=\frac{\alpha_{\mathrm{toa}}-\alpha_{\mathrm{p}}}{\tau_{\mathrm{sw}}{ }^{2}}$

em que $\alpha_{T O A}$ é o albedo planetário e $\alpha_{p}$ é a porção média da radiação solar refletida pela atmosfera terrestre antes de alcançar a superfície, que varia entre 0,025 e 0,040, e $\tau_{s w}$ é a transmissividade atmosférica para onda curta. $\mathrm{Na}$ aplicação do algoritmo SEBAL, é recomendado o uso do valor de $\alpha_{p}=0,03$ (BASTIAANSSEN, 2000).

O Índice de Vegetação da Diferença Normalizada (Normalized 
PAVÃO, V. M.; QUERINO, C. A. S.; BENEDITTI, C. A.; PAVÃO, L. L.; QUERINO, J. K. A. S.; MACHADO, N. G.; BIUDES, M. S.

VARIAÇÃO ESPACIAL E TEMPORAL DO SALDO DE RADIAÇÃO SUPERFICIAL EM UMA ÁREA DO SUL DO AMAZONAS, BRASIL

Difference Vegetation Index - NDVI) foi calculado pela Equação (7).

$\mathrm{NDVI}=\frac{\rho_{\mathrm{IV}}-\rho_{\mathrm{V}}}{\rho_{\mathrm{IV}}+\rho_{\mathrm{V}}}$

em que $\rho_{\mathrm{IV}}$ e $\rho_{\mathrm{V}}$ correspondem às bandas 4 e 3 do TM Landsat 5, respectivamente.

O Índice de Vegetação Ajustado ao Solo (soil-adjusted vegetation índex SAVI) foi calculado pela Equação (8). O SAVI é um índice que busca amenizar os efeitos do "background" do solo.

SAVI $=\frac{(1+L)\left(\rho_{\mathrm{IV}}-\rho_{\mathrm{V}}\right)}{\left(L+\rho_{\mathrm{IV}}+\rho_{\mathrm{V}}\right)}$

em que $L$ é uma constante de ajuste do SAVI. Nesse trabalho foi utilizado $L=$ 0,5 (ALLEN et al., 2007).

O Índice de área Foliar (IAF) é a razão entre a área foliar de toda a vegetação por unidade de área projetada pela mesma vegetação. O IAF mostra-se com um indicador da biomassa de cada pixel da imagem e o mesmo foi computado pela Equação (9) (ALLEN et al., 2002).

$\mathrm{IAF}=-\frac{\ln \left(\frac{0,69-\mathrm{SAVI}}{0,59}\right)}{0,91}$

A temperatura da superfície foi estimada com base na equação de Planck invertida, proposta para um corpo negro. A temperatura na superfície $\left(T_{s}\right.$, em K) foi calculada pela Equação (10), em função da radiância espectral da banda termal $L_{\lambda 6}$ do TM Landsat 5 e da emissividade ( $\varepsilon N B$ ).

$$
\mathrm{T}_{\mathrm{s}}=\frac{\mathrm{K}_{2}}{\ln \left(\frac{\varepsilon_{\mathrm{NB}} \mathrm{K}_{1}}{\mathrm{~L}_{\lambda, 6}}+1\right)}
$$

em que $\mathrm{K}_{1}=607,76 \mathrm{~W} \mathrm{~m}^{-2} \mathrm{sr}^{-1} \mu \mathrm{m}^{-1}$ e $\mathrm{K}_{2}=1260,56 \mathrm{~K}$ são constantes de calibração da banda termal do TM Landsat 5 (ALLEN et al., 2002).

Devido ao fato de cada pixel não emitir energia eletromagnética como um corpo negro, há a necessidade de estabelecer sua emissividade no domínio espectral da banda termal imagiada pelo canal termal $(\varepsilon N B)$, obtida pela 
PAVÃO, V. M.; QUERINO, C. A. S.; BENEDITTI, C. A.; PAVÃO, L. L.; QUERINO, J. K. A. S.; MACHADO, N. G.; BIUDES, M. S.

VARIAÇÃO ESPACIAL E TEMPORAL DO SALDO DE RADIAÇÃO SUPERFICIAL EM UMA ÁREA DO SUL DO AMAZONAS, BRASIL

Equação (11) (ALLEN et al., 2002).

$\varepsilon \mathrm{NB}=0,97+0,00331 \mathrm{IAF}$

em que IAF é o Índice de área foliar $\left(\mathrm{m}^{2} \mathrm{~m}^{-2}\right)$. Considerou-se $\varepsilon N B=0,98$ quando IAF > 3 e $\varepsilon N B=0,99$ para corpos de água (ALLEN et al., 2002).

$A$ radiação de onda longa emitida pela superfície $R_{o l, e m i}\left(W m^{-2}\right)$ foi obtida pela equação de Stefan-Boltzman (Equação 12).

$\mathrm{R}_{\mathrm{ol}, \mathrm{emi}}=\varepsilon_{0 . \sigma} \cdot \mathrm{T}_{\text {sup }}{ }^{4}$

em que $\varepsilon_{0}$ é a emissividade da superfície em cada pixel, $\sigma$ é a constante de Stefan-Boltzman $\sigma=5,67.10^{8} \mathrm{~W} \mathrm{~m}^{-2} \mathrm{~K}^{-4}$ e $\mathrm{T}_{\text {sup }}$ é a temperatura da superfície $(\mathrm{K})$.

A radiação de onda longa emitida pela atmosfera na direção da superfície

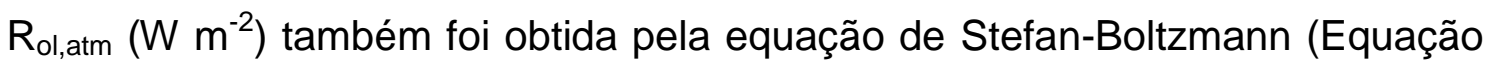
13) em função da temperatura do $\operatorname{ar}\left(T_{a}\right.$, em $\left.K\right)$ e da emissividade atmosférica obtida $\left(\varepsilon_{\mathrm{a}}\right)$, dada por $\varepsilon_{\mathrm{a}}=0,85 .\left(-\ln \tau_{\mathrm{sw}}\right)^{0,09}$ (ALLEN et al., 2002).

$\mathrm{Rol}_{\mathrm{atm}}=\varepsilon_{\mathrm{a}} \cdot \sigma \cdot \mathrm{T}_{\mathrm{a}}{ }^{4}$

$A$ radiação de onda curta incidente $R_{\text {sol,inc }}\left(W^{-2}\right)$ é o fluxo de radiação solar direta e difusa que atinge a superfície terrestre. $A R_{\text {sol,inc }}$ foi estimada pela Equação (14), assumindo a condição de céu claro (ALLEN et al., 2002).

$\mathrm{R}_{\mathrm{sol}, \text { inc }}=\mathrm{S} \cdot \cos \mathrm{Z} \cdot \mathrm{d}_{\mathrm{r} . \tau_{\mathrm{sw}}}$

em que S é a constante solar $\left(1367 \mathrm{Wm}^{-2}\right), \mathrm{Z}$ é ângulo zenital solar, $\mathrm{d}_{\mathrm{r}}$ é o inverso do quadrado da distância relativa Terra-Sol e $\tau_{\mathrm{sw}}$ é a transmissividade atmosférica. $A R_{\text {sol,inc }}$ foi considerada constante em toda a área de estudo.

Por fim, o saldo de radiação da superfície $\left(R_{n}\right.$, em $\left.W m^{-2}\right)$ foi estimado pela Equação 15.

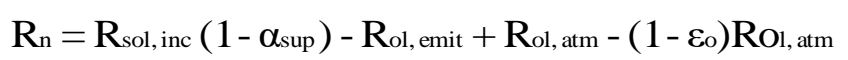

\section{RESULTADOS E DISCUSSÃO}

\subsection{Variação Espaço-Temporal do NDVI e SAVI}

Na Figura 2 é observado valores de NDVI e SAVI para o quadrante da 
PAVÃO, V. M.; QUERINO, C. A. S.; BENEDITTI, C. A.; PAVÃO, L. L.; QUERINO, J. K. A. S.; MACHADO, N. G.; BIUDES, M. S.

VARIAÇÃO ESPACIAL E TEMPORAL DO SALDO DE RADIAÇÃO SUPERFICIAL EM UMA ÁREA DO SUL DO AMAZONAS, BRASIL

área de estudo em 15/07/2009 e 02/07/2010. Os tons de verde correspondem a vegetação mais densa, o verde escuro com valores superiores a 0,7 indica uma vegetação sadia com pleno crescimento ativo, com baixa refletância na faixa do vermelho, e alta refletância no infravermelho próximo. Valores elevados de NDVI e SAVI também indicam maior disponibilidade de água no solo que proporciona melhores condições de desenvolvimento vegetativo para as plantas (FILHO et al., 2007; BORATTO e GOMIDE, 2013).
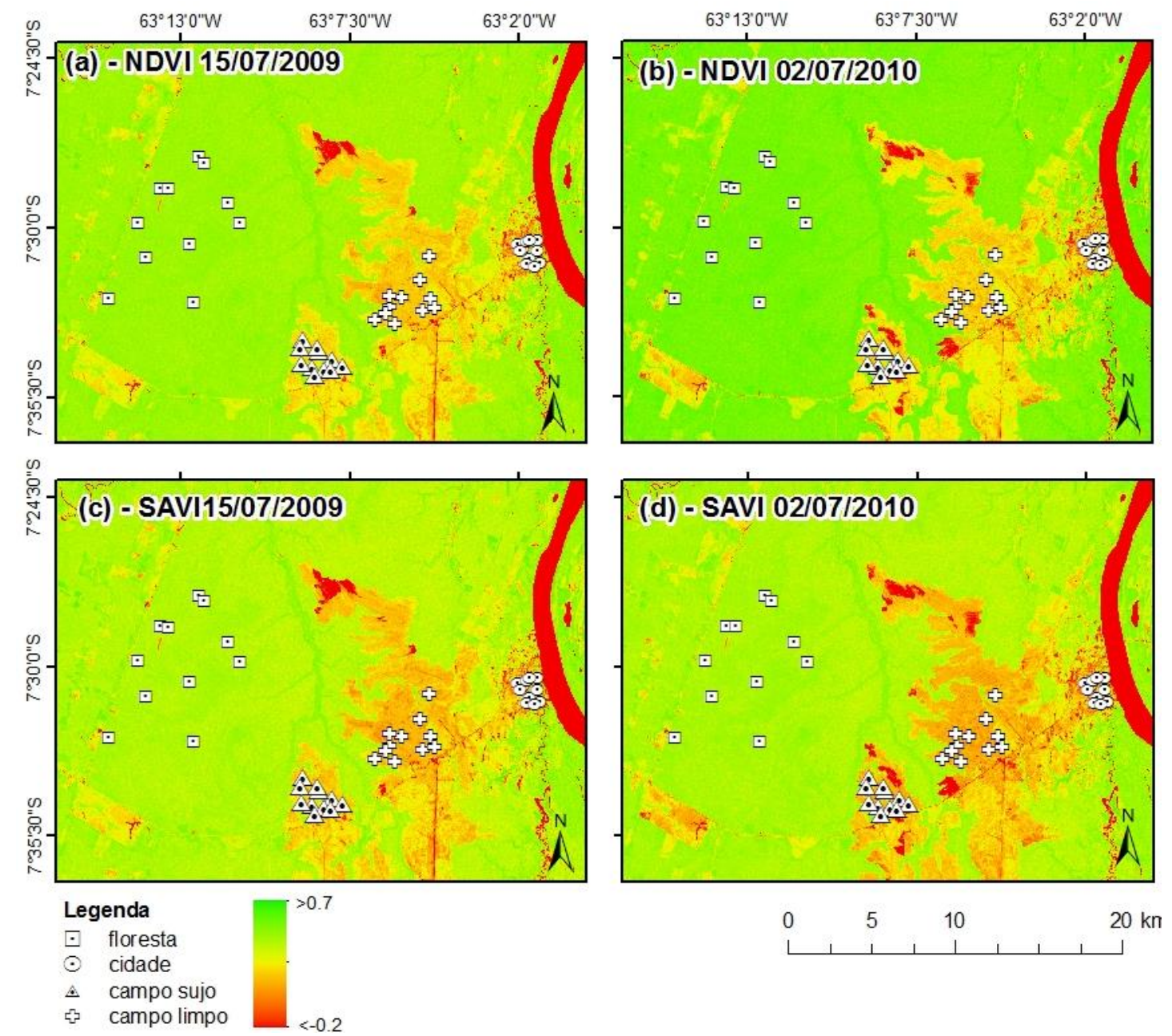

Figura 2: Índice de Vegetação da Diferença Normalizada (NDVI) e Índice de Vegetação Ajustado ao Solo (SAVI) para 15/07/2009 e 02/07/2010 em diferentes uso do solo no município de Humaitá-AM.

A Figura 3 apresenta a variação do NDVI e SAVI entre as áreas de floresta, campo sujo, campo limpo e cidade durante o período estudado. Os dois índices apresentaram variações semelhantes nas duas datas de estudo, 
PAVÃO, V. M.; QUERINO, C. A. S.; BENEDITTI, C. A.; PAVÃO, L. L.; QUERINO, J. K. A. S.; MACHADO, N. G.; BIUDES, M. S.

VARIAÇÃO ESPACIAL E TEMPORAL DO SALDO DE RADIAÇÃO SUPERFICIAL EM UMA ÁREA DO SUL DO AMAZONAS, BRASIL

demostrando baixa variação anual. Os maiores valores de NDVI e SAVI ocorreram em áreas de floresta, com valores superiores a 0,6 e 0,5, respectivamente. Ambientes de campos e cidade não apresentaram diferença, contudo verificou-se tendência de menores valores ocorrendo em área de campo limpo e cidade (entre 0,3 e 0,45) quando comparadas com campo sujo (entre 0,4 e 0,5$)$.
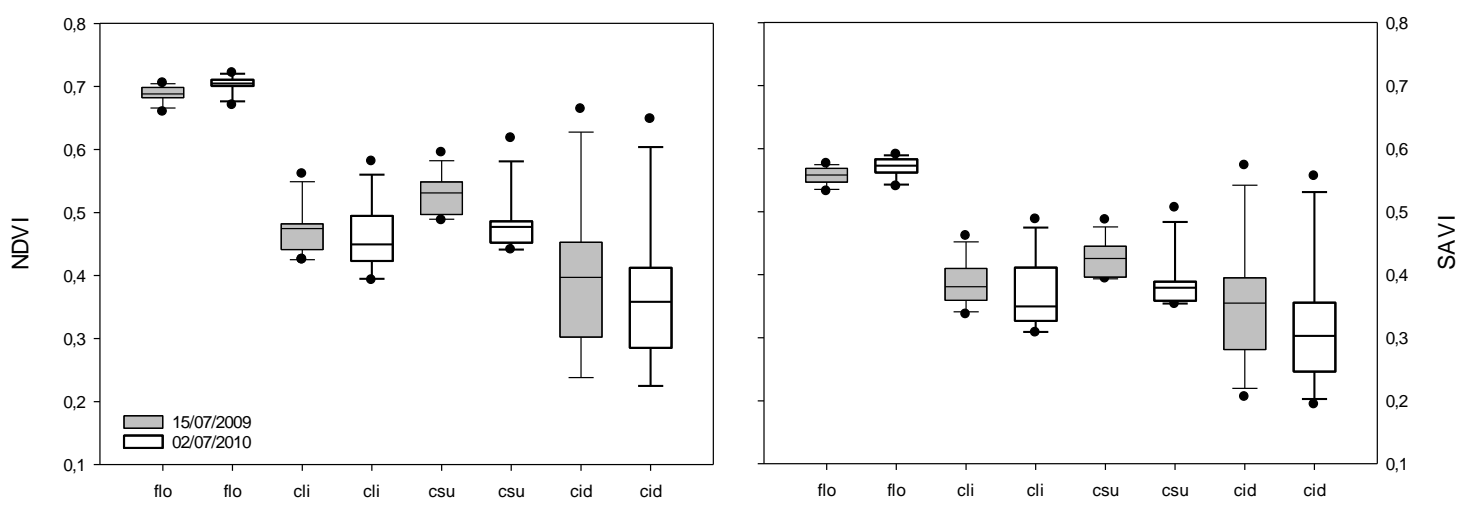

Uso do solo

Figura 3: Variação de NDVI (a) e SAVI (b) nos ambientes de floresta (flo), campo limpo (cli), campo sujo (csu) e cidade (cid) no município de Humaitá-AM em 15/07/2009 e 02/07/2010.

Os maiores valores de NDVI e SAVI na floresta foram devido à maior altura das árvores e densidade do dossel. Nessa região, áreas de floresta apresentam densidade arbórea de 930 ind/ha e área basal de $19,2 \mathrm{~m}^{2} / \mathrm{ha}$, enquanto que no campo esses valores são de $430 \mathrm{ind} / \mathrm{hac}$ e $9,2 \mathrm{~m}^{2} / \mathrm{ha}$, respectivamente (VIDOTTO et al., 2007). As áreas de campo limpo apresentam vegetação predominantemente herbácea com poucos arbustos e inexistência de arvores. Por outro lado, as áreas de campo sujo apresentam uma mistura de solo e pouca cobertura arbórea, com presença de arbustos e ervas (VIDOTTO et al., 2007).

Ressalta-se que, valores de NDVI e SAVI inferiores a zero indicam presença de água ou núvem (BORATTO e GOMIDE, 2013), como é possível notar nas áreas correspondentes ao Rio Madeira (Figura 2). Valores próximos a -1 indicam maior qualidade da água, maior profundidade e ausência de plantas aquáticas (FAUSTO et al., 2014). O intervalo compreendido entre 0,3 - 
PAVÃO, V. M.; QUERINO, C. A. S.; BENEDITTI, C. A.; PAVÃO, L. L.; QUERINO, J. K. A. S.; MACHADO, N. G.; BIUDES, M. S.

VARIAÇÃO ESPACIAL E TEMPORAL DO SALDO DE RADIAÇÃO SUPERFICIAL EM UMA ÁREA DO SUL DO AMAZONAS, BRASIL

0,4 indica áreas de solo exposto, sendo observada principalmente em campo limpo, a qual vai diminuindo gradativamente à medida em que a vegetação se torna mais densa.

\subsection{Saldo de radiação}

A Figura 4 apresenta a distribuição espacial do Saldo de Radiação $(R n)$ em 15/07/2009 e 02/07/10 na área de estudo. O Rn variou de valores inferiores a 300 W.m $\mathrm{m}^{-2}$ (azul escuro) a valores superiores a $600 \mathrm{~W} . \mathrm{m}^{-2}$ (vermelho). A ocorrência dos maiores valores de $\mathrm{Rn}$ tenderam a acompanhar as áreas com maiores valores de NDVI e SAVI, exceto quando da presença de água, a qual apresenta baixa refletância em praticamente todo 0 espectro solar, consequentemente a energia absorvida nesses locais será alta (PEREIRA et al., 2007).
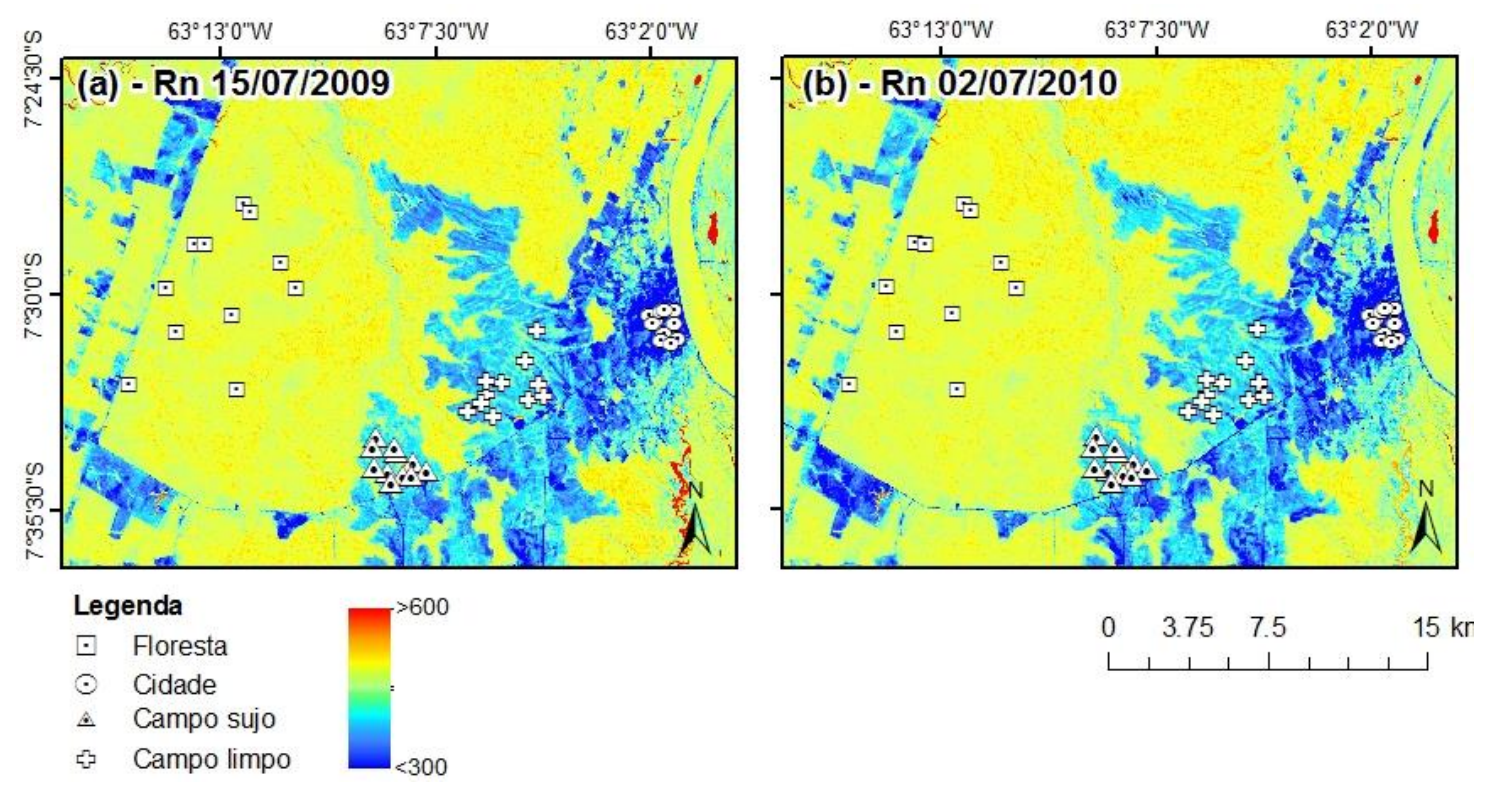

Figura 4: Distribuição espacial do Saldo de Radiação para as cenas 15/07/2009 (a) e 02/07/2010 (b) no município de Humaitá - AM.

Os maiores valores de Rn ocorreram em áreas de floresta, enquanto que os menores ocorreram em áreas modificadas (incluindo área urbana). O Rn 
PAVÃO, V. M.; QUERINO, C. A. S.; BENEDITTI, C. A.; PAVÃO, L. L.; QUERINO, J. K. A. S.; MACHADO, N. G.; BIUDES, M. S.

VARIAÇÃO ESPACIAL E TEMPORAL DO SALDO DE RADIAÇÃO SUPERFICIAL EM UMA ÁREA DO SUL DO AMAZONAS, BRASIL

médio na floresta foi aproximadamente 550 e $565 \mathrm{~W} . \mathrm{m}^{-2}$ nos dias 15/07/2009 e 02/07/2010, respectivamente (Figura 5). Esses valores foram próximos aos encontrados em uma área de floresta sobre a reserva biológica de Jaru em Rondônia (OLIVEIRA et al., 2003). O Rn médio no campo sujo foi $526 \mathrm{~W} \mathrm{~m}^{-2}$ em 15/07/2009 e $535 \mathrm{~W} \mathrm{~m}^{-2}$ em 02/07/2009. Valores similares aos encontrados no campo sujo desse trabalho foram observados em cerrado amazônico (AMANAJÁS et al., 2013). O Rn médio nas áreas modificadas, incluindo a zona urbana, apresentou valores inferiores a $500 \mathrm{~W} . \mathrm{m}^{-2}$, corroborado por Andrade et al. (2014), que encontraram valores entre 472 e $523 \mathrm{~W} \cdot \mathrm{m}^{-2}$ em área urbana no Pará.

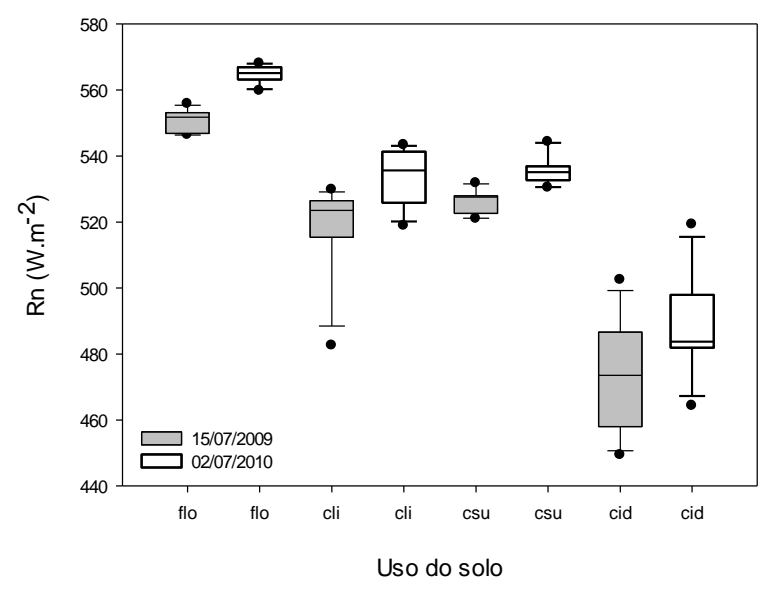

Figura 5: Comportamento do Rn em ambientes de floresta (flo), campo limpo (cli), campo sujo (csu) e cidade (cid) no município de Humaitá-AM em 15/07/2009 e 02/07/2010.

Os maiores valores de $\mathrm{Rn}$ na floresta e menores em áreas modificadas foram devido à diferenças óticas da superfície que, por conseguinte, provocam variações no albedo da superfície. Como o $\mathrm{Rn}$ representa a soma dos balanços de onda curta e longa na superfície, a energia disponível no ambiente varia em função da radiação solar, do albedo e da temperatura da superfície e da atmosfera. Em um mesmo dia de céu claro, como os dias desse estudo, o Rn varia principalmente devido à variação do albedo que é função do verdor da superfície (OLIVEIRA e MORAIS, 2013; AMANAJÁS et al., 2013). Portanto, há uma correlação de caráter inverso entre $\mathrm{Rn}$ e albedo, onde áreas com maiores valores de albedo estão associadas às áreas com menores Rn (OLIVEIRA e 
PAVÃO, V. M.; QUERINO, C. A. S.; BENEDITTI, C. A.; PAVÃO, L. L.; QUERINO, J. K. A. S.; MACHADO, N. G.; BIUDES, M. S.

VARIAÇÃO ESPACIAL E TEMPORAL DO SALDO DE RADIAÇÃO SUPERFICIAL EM UMA ÁREA DO SUL DO AMAZONAS, BRASIL

MORAIS, 2013). O que explica a ocorrência de maior absorção da radiação solar incidente em áreas de floresta quando comparadas com áreas de pastagem (SANTOS et al., 2014).

\subsection{Relação entre Rn, NDVI e SAVI}

A Figura 6 apresenta a correlação positiva dos índices NDVI e SAVI e $\mathrm{Rn}$ nos diferentes tipos de cobertura do solo nas datas analisadas, com maiores valores de $\mathrm{Rn}$ em áreas de florestas, que apresentam maiores índices de vegetação, e menores valores de $\mathrm{Rn}$ na cidade, onde os índices de vegetação são baixos.

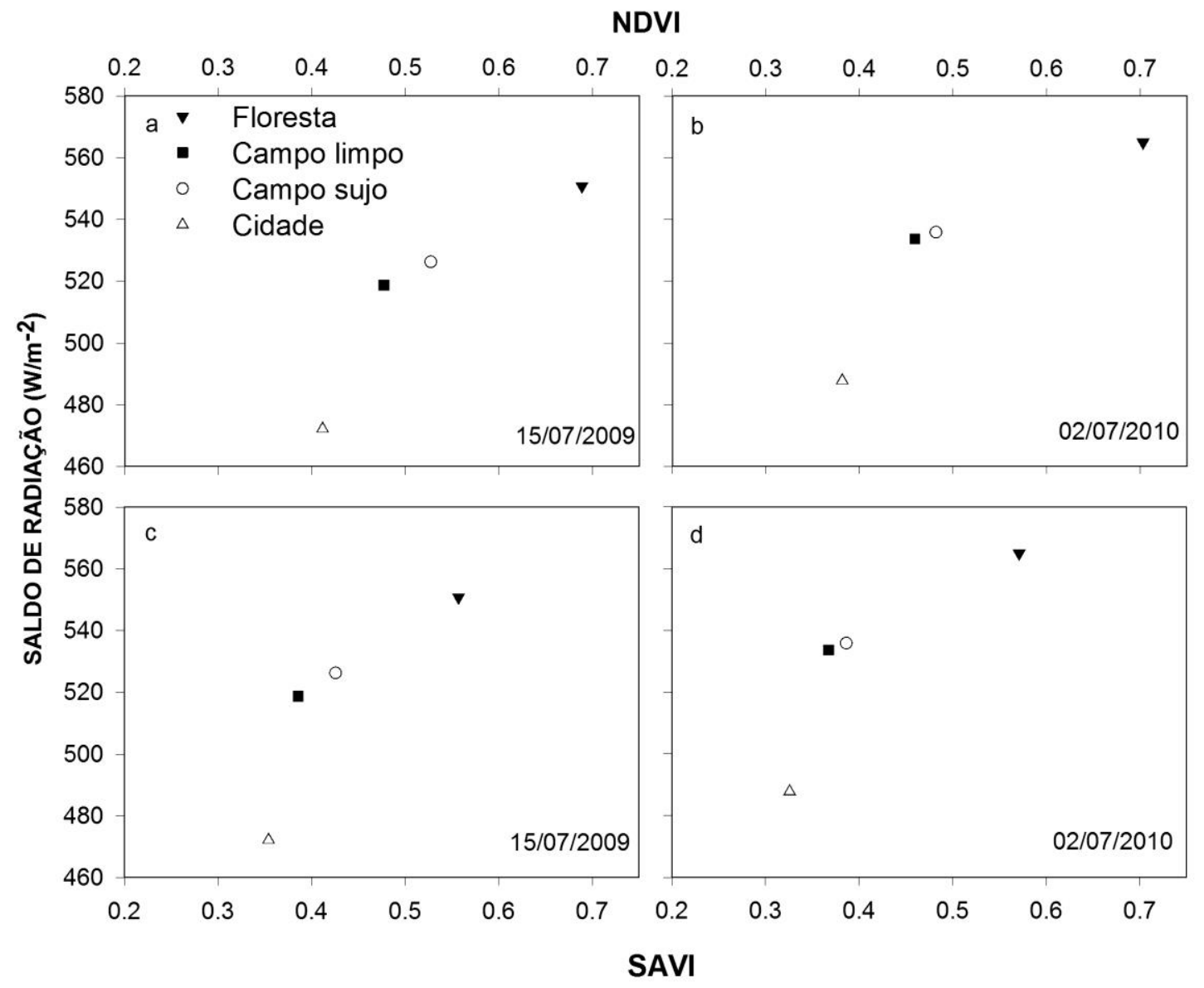

Figura 6: Relação entre Rn e NDVI (a e b) e Rn e SAVI (c e d) em diferentes coberturas do solo no município de Humaitá - AM nos dias 15/07/2009 e 02/07/2010. 
Os menores valores de $\mathrm{Rn}$ em áreas de campo e cidade são devido à baixa densidade de vegetação, demonstrada pelos menores valores dos índices de vegetação. Nessas áreas, há maior reflexão da radiação solar, que diminui o balanço de ondas curtas. No entanto, em ambientes de floresta, devido à sua maior densidade de folhas e galhos, boa parte dessa radiação é utilizada para os processos fotossintéticos. Além disso, a folhagem na floresta é agrupada à copa das árvores, com picos e depressões organizadas na superfície dos dosséis. Assim, a maior parte da radiação solar incidente penetra no interior da floresta antes de ser refletida, resultando numa acentuada captura de radiação solar e, consequentemente, aumento do balanço de ondas curtas (LEITÃO et al., 2002).

A evapotranspiração, definida como a perda de água das plantas como vapor dentro da atmosfera, consome a maior parte da energia disponível ao ambiente, provocando diminuição do calor sensível e consequentemente resfriamento da superfície vegetada e do ar nos seus arredores. A diminuição da temperatura da superfície contribui para aumento do balanço de ondas longas, e dessa forma, para o aumento do Rn (FAUSTO et al., 2014).

\section{CONCLUSÃO}

A heterogênea cobertura vegetal e à existência de áreas urbanas provocou uma elevada variação espacial nos índices de vegetação e, consequentemente no saldo de radiação na área de estudo do sul do Amazonas.

Apesar da área de estudo desse trabalho ser restrita a uma pequena parte do sul do Amazonas, os resultados evidenciam o efeito do desmatamento na diminuição da energia disponível ao ecossistema (saldo de radiação), a qual contribui para alteração no balanço de energia e água no sistema solo-plantaatmosférica.

\section{AGRADECIMENTOS}


PAVÃO, V. M.; QUERINO, C. A. S.; BENEDITTI, C. A.; PAVÃO, L. L.; QUERINO, J. K. A. S.; MACHADO, N. G.; BIUDES, M. S.

VARIAÇÃO ESPACIAL E TEMPORAL DO SALDO DE RADIAÇÃO SUPERFICIAL EM UMA ÁREA DO SUL DO AMAZONAS, BRASIL

A pesquisa foi apoiada pela Universidade Federal de Mato Grosso (UFMT), Programa de Pós-Graduação em Física Ambiental (PPGFA/IF/UFMT), e Coordenação de Aperfeiçoamento de Pessoal do Ensino Superior (CAPES Bolsas, Processo no 9750/13-4 e no 9768/13-0), Conselho Nacional de Desenvolvimento Científico e Tecnológico (CNPq - Bolsa, Processo no 303625/2015-5), e financiada pela Fundação de Amparo à Pesquisa do Estado de Mato Grosso (FAPEMAT - PRONEM 2014, processo ํㅜ 561397/2014).

\section{REFERÊNCIAS}

ALLEN, R. G.; TASUMI, M.; TREZZA, R. Satellite-Based energy balance for mapping evapotraspiration with internalized calibration (METRIC) - Model. Journal of Irrigation and Drainage Engineering, v.133, n.4, p.380-394, 2007.

ALLEN, R. G.; TASUMI, M.; TREZZA, R;. Waters, R.; Bastiaanssen, W. Surface Energy Balance Algorithms for Land (SEBAL). Advanced Training and users Manual, Kimberly, Idaho, v.1,0, 97p. 2002.

AMANAJÁS, J. C.; BRAGA, C. C.; ALCÂNTARA.; C. R.; SOUSA, L. F.; OLIVEIRA5, G.B.. Estimativa do saldo de radiação à superfície em área de cerrado amazônico utilizando imagem Landsat 5 - TM. In: Anais XVI Simpósio Brasileiro de Sensoriamento Remoto, Foz do Iguaçu, Sociedade Brasileira de Sensoriamento Remoto, 2013.

ANDRADE, S. C. P.; CORRÊA, J. A. J. Estimativa do saldo de radiação instantâneo à superfície para a cidade de Santarém-PA, através de imagens do Landsat 5-TM. Revista Brasileira de Geografia Física, v.7, n.4, 653-661. 2014.

BASTIAANSSEN, W. G. M. Sebal based sensible and latent heat fluxes in the irrigated Gediz Basin, Turkey. Journal of Hidrology, v.229, p.87-100, 2000.

BIUDES, M. S.; CAMPELO JUNIOR, J. H.; NOGUEIRA, J. S.; SANCHES, L. Estimativa do balanço de energia em cambarazal e pastagem no norte do Pantanal pelo método de razão de Bowen. Revista Brasileira de Meteorologia, v.24, n.2, p. 135-143, 2009. 
PAVÃO, V. M.; QUERINO, C. A. S.; BENEDITTI, C. A.; PAVÃO, L. L.; QUERINO, J. K. A. S.; MACHADO, N. G.; BIUDES, M. S.

VARIAÇÃO ESPACIAL E TEMPORAL DO SALDO DE RADIAÇÃO SUPERFICIAL EM UMA ÁREA DO SUL DO AMAZONAS, BRASIL

BIUDES, M. S.; VOULITIS, G. L.; MACHADO, N. G.; DE ARRUDA, P. H. Z.; NEVES, G. A. R.; LOBO, F. A.; NEALE, C. M. U.; NOGUEIRA, J. S. Patterns of energy exchange for tropical ecosystems across a climate gradiente in Mato Grosso, Brazil. Agricultural and Forest Meteorology, v.202, p.112-124, 2015.

BORATTO, I. M. P.; GOMIDE, R. L. Aplicação dos índices de vegetação NDVI, SAVI e IAF na caracterização da cobertura vegetativa da região Norte de Minas Gerais. 2013. In: Anais XVI Simpósio Brasileiro de Sensoriamento Remoto, Foz do Iguaçu, Sociedade Brasileira de Sensoriamento Remoto, 2013.

BRAUN, E.H.G.; RAMOS, J.R.A. Estudo agroecológico dos campos PuciaríHumaitá (Estado do Amazonas e Território Federal de Rondônia). Revista Brasileira de Geografia, v.21, p.443-497, 1959.

CAMPOS, M. C. C. Pedogeomorfologia aplicada a ambientes amazônicos do médio Rio Madeira. 224 p. Tese (Doutorado em Ciência do Solo) Universidade Federal Rural de Pernambuco, Recife. 2009.

CAMPOS, M. C. C.; RIBEIRO, M. R.; JUNIOR, V. S. S.; FILHO, R. R. M.; ALMEIDA, M. C. Topossequência de solos na transição Campos NaturaisFloresta na região de Humaitá, Amazonas. Acta Amazônica, Manaus-AM, v.42, n.3, p.387-398. 2012.

CHANDER, G., MARKHAM, B. L., \& BARSI, J. A. Revised Landsat 5 Thematic Mapper radiometric calibration. IEEE Transactions on Geoscience and Remote Sensing, v.44, p.490-494. 2007.

DEMARCHI, J. C.; PIROLI, E. L.; ZIMBACK, C. R. L. Analise temporal do uso do solo e comparação entre os índices de vegetação NDVI e SAVI no município de Santa Cruz Do Rio Pardo - SP usando imagens LANDSAT-5. RA'E GA, v.21, p.234-271, 2011.

ELMORE, A. J.; MUSTARD, J. F.; MANNING, S. J.; LOBELL, D. B. Quantifying Vegetation Change in Semiarid Environments: Precision and Accuracy of Spectral Mixture Analysis and the Normalized Difference Vegetation Index. 
PAVÃO, V. M.; QUERINO, C. A. S.; BENEDITTI, C. A.; PAVÃO, L. L.; QUERINO, J. K. A. S.; MACHADO, N. G.; BIUDES, M. S.

VARIAÇÃO ESPACIAL E TEMPORAL DO SALDO DE RADIAÇÃO SUPERFICIAL EM UMA ÁREA DO SUL DO AMAZONAS, BRASIL

Remote Sensing of Environment, v.73, n.1, p.87-102, 2000.

FAUSTO, M. A.; MACHADO, N. G.; NOGUEIRA, J. S.; BIUDES, M. S. Net radiation estimated by remote sensing in Cerrado areas in the Upper Paraguay River Basin. Journal of Applied Remote Sensing, v.8, p.083541, 2014.

FERREIRA, L. V.; VENTICINQUE, E.; ALMEIDA, S. O desmatamento na Amazônia e a importância das áreas protegidas. Estudos avançados, v.19, n.53, p.157-166, 2005.

FILHO, J. F. C.; FRANCISCO, P. R. M.; ANDRADE, M. V.; SILVA, L.; DANTAS, L. M. Estimativa do índice de vegetação da diferença normalizada (NDVI) na Microrregião de Sousa-PB utilizando imagens do CBERS-21. 2007. In: Congresso Brasileiro de Agrometeorologia, Aracaju, Sociedade Brasileira de Agrometeorologia, 2007.

GUSMÃO, A. C. V. L.; SILVA, B. B. MONTENEGRO, S.M. G. L.; GALVÍNCIO, J. D. Determinação do saldo radiativo na Ilha do Bananal, TO, com imagens orbitais. Revista Brasileira de Engenharia Agrícola e Ambiental. v.16, n.10, p.1107-1114, 2012.

HUETE, A. R., 1988. A soil-adjusted vegetation index. Remote Sensing of Environment, v.25, p.295-309.

IQBAL, M. An introduction to solar radiation. New York: Academic Press. 1983, 212p.

LEITÃO, M. M. V. B. R.; Santos E Oliveira. Estimativas do albedo em três ecossistemas da floresta amazônica. Revista Brasileira de Engenharia Agrícola e Ambiental, v.6, n.2, p.256-261. 2002.

LIBERATO, A. M. SILVA, B. B. CARDOSO, F. L. Uso de técnicas de sensoriamento remoto na estimativa do saldo de radiação em Rondônia. Revista Pesquisa \& Criação, v.10, n.2, p.153-164, 2011 
PAVÃO, V. M.; QUERINO, C. A. S.; BENEDITTI, C. A.; PAVÃO, L. L.; QUERINO, J. K. A. S.; MACHADO, N. G.; BIUDES, M. S.

VARIAÇÃO ESPACIAL E TEMPORAL DO SALDO DE RADIAÇÃO SUPERFICIAL EM UMA ÁREA DO SUL DO AMAZONAS, BRASIL

MARKHAM, B. L.; BARKER, J. L. Thematic mapper bandpass solar exoatmospherical irradiances. International. Journal of Remote Sensing, v.8, n.3, p.517-523, 1987.

MARKHAM, B. L.; BARKER, J. L. Thematic mapper bandpass solar exoatmospherical irradiances. International. Journal of Remote Sensing, v.8, n.3, p.517-523, 1987.

MARTINS, G. C. FERREIRA, M. M. CURI, N. VITORINO, A. C. T.; SILVA, M. L. N. Campos nativos e matas adjacentes da região de Humaitá (AM): atributos diferenciais dos solos. Ciência e Agrotecnologia, v.30, n.2, p.221-227, 2006.

OLIVEIRA, G.; MORAES, E. C. Validação do balanço de radiação obtido a partir de dados MODIS/TERRA na Amazônia com medidas de superfície do LBA. Revista ACTA Amazônica, v.43, n.3, p.353-364, 2013.

OLIVEIRA, G.; MORAES, E. C.; SHIMABUKURO, Y. E.; RUDORFF, B. F. T.; ALVALÁ, R. C. S.; SANTOS, T. V. Avaliação do albedo em diferentes tipos de uso e cobertura da terra no sudoeste da Amazônia. 2013. In: Anais XVI Simpósio Brasileiro de Sensoriamento Remoto, Foz do Iguaçu, Sociedade Brasileira de Sensoriamento Remoto, 2013.

PEREIRA, G.; MORAES, E. C.; ARAI, E. OLIVEIRA, L. G. L. Estudo preliminar da estimativa da reflectância e albedo de microssistemas pantaneiros a partir de imagens de satélite. Revista Brasileira de Cartografia, v.59, n.1, p.55-61, 2007.

SANTOS, C. A. C.; WANDERLEY, R. L. N.; ARAÚJO, A. L.; BEZERRA, B. G. Obtenção do saldo de radiação em áreas de pastagem e floresta na Amazônia (estação seca) através do sensor MODIS. Revista Brasileira de Meteorologia, v.29, n.3, 420-432, 2014.

SILVA, B. B.; LOPES, G. M.; AZEVEDO, P. V. BALANÇO DE RADIAÇÃO EM ÁREAS IRRIGADAS UTILIZANDO IMAGENS LANDSAT 5 - TM. Revista Brasileira de Meteorologia, v.20, n.2, 243-252, 2005. 
PAVÃO, V. M.; QUERINO, C. A. S.; BENEDITTI, C. A.; PAVÃO, L. L.; QUERINO, J. K. A. S.; MACHADO, N. G.; BIUDES, M. S.

VARIAÇÃO ESPACIAL E TEMPORAL DO SALDO DE RADIAÇÃO SUPERFICIAL EM UMA ÁREA DO SUL DO AMAZONAS, BRASIL

VIDOTTO, E.; PESSENDA, L.C. R.; RIBEIRO, A. S.; FREITAS, H. A.; BENDASSOLLI, J. A. Dinâmica do ecótono floresta-campo no sul do estado do Amazonas no Holoceno, através de estudos isotópicos e fitossociológicos. Acta Amazônica, v.37, n.3, p.385-400, 2007.

YANAI, A. M.; FEARNSIDE, P. M.; GRAÇA, P. M. L. A. Desmatamento no sul do Amazonas: simulação do efeito da criação da Reserva de Desenvolvimento Sustentável do Juma. 2011. In: Anais XVI Simpósio Brasileiro de Sensoriamento Remoto, Curitiba, Sociedade Brasileira de Sensoriamento Remoto, 2011. 